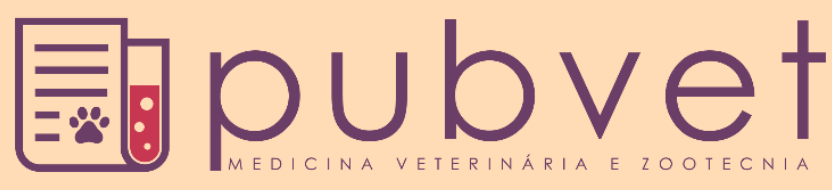

https://doi.org/10.31533/pubvet.v13n11a445.1-9

\title{
Estase e obstrução gastrointestinal em coelhos domésticos (Oryctolagus cuniculus): revisão
}

\author{
Natália Pitanguy de Almeida Meneses ${ }^{1}$, Leonardo Toshio Oshio ${ }^{2} \bullet$, Anna Marcella Neves Dias ${ }^{3} \bullet$ \\ ${ }^{I}$ Discente no Curso de Medicina Veterinária do Centro Universitário Presidente Antônio Carlos - UNIPAC - Juiz de Fora - MG - \\ ${ }^{2}$ Orientador, Doutor, Médico Veterinário e Docente no Curso de Medicina Veterinária do Carlos - UNIPAC - Juiz de Fora - MG \\ ${ }^{3}$ Docente no Curso de Medicina Veterinária do Centro Universitário Presidente Antônio Carlos - UNIPAC-Juiz de Fora - MG \\ *Autor para correspondência, E-mail: natypitanguy_@hotmail.com
}

Resumo. Com o crescente número de coelhos como animais de estimação, os chamados pets não convencionais são de extrema importância que o médico veterinário esteja a par das principais afecções desses animais, bem como o seu manejo alimentar e ambiental. Estase e obstrução gastrointestinal são algumas das principais doenças que afetam esse animal, caracterizadas pela diminuição ou ausência dos pellets fecais, anorexia, dor abdominal, desconforto à palpação, sendo consideradas emergenciais, requerendo rapidez no atendimento, no diagnostico diferencial, pois demonstram sinais iguais e tratamentos diferentes, e na terapia a ser instituída, podendo ser um desafio para o médico veterinário, pois tem o tratamento complexo e resposta terapêutica lenta. Esta revisão teve como objetivo abordar sobre a estase e a obstrução gastrointestinal em coelhos domésticos.

Palavras chave: anorexia, dor, gastrointestinal, hipomotilidade, lagomorfo, peristaltismo

\section{Gastrointestinal stasis and obstruction in domestic rabbits (Oryctolagus cuniculus): review}

\begin{abstract}
With the increasing number of rabbit's pets, the so called non-conventional type, it is extremely important that all veterinary doctors are aware of its specific illness, and also its environmental and feeding management. Gastrointestinal stasis and obstruction are some of its main diseases, characterized by reduction or absence of fecal pellets, anorexic, abdominal pain, palpation discomfort, all considered emergencies to be dealt with utmost priority and quickness, both in differential diagnosis and therapy, as most of the clinical signals are similar, but have different approaches, which proves to be a challenge for the veterinary doctor with its complex therapy and slow results. This revision objective was to approach the gastrointestinal stasis and obstruction theme in domestic rabbits.
\end{abstract}

Keywords: anorexia, pain, gastrointestinal, hypomotility, lagomorph, peristalsis

\section{Estasis y obstrucción gastrointestinal en conejos domésticos (Oryctolagus cuniculus): revisión}

Resumen. Con el creciente número de conejos como mascotas, las llamadas mascotas no convencionales, es extremadamente importante que el veterinario sea consciente de las principales afecciones de estos animales, así como de su alimentación y el manejo ambiental. La estasis y la obstrucción gastrointestinal son algunas de las principales enfermedades que afectan a este animal, caracterizadas por la disminución o ausencia de gránulos fecales, anorexia, dolor abdominal, molestias de palpación y se consideran de emergencia, lo que requiere rapidez en la atención, diagnóstico diferencial, ya que muestran 
signos iguales diferentes tratamientos, y la terapia a instituir, que puede ser un desafío para el médico veterinario, ya que tiene un tratamiento complejo y una respuesta terapéutica lenta. Esta revisión tuvo como objetivo tratar el tema de estasis y obstrucción gastrointestinal en conejos domésticos.

Palabras clave: anorexia, dolor, gastrointestinal, hipomotilidad, lagomorfo, peristaltismo

\section{Introdução}

Os coelhos domésticos (Oryctolagus cuniculus) têm se tornado cada vez mais animais de companhia, havendo uma crescente demanda por atendimento a estes animais chamados "pets não convencionais". É comum que estes apresentem problemas como bolas de pelo no estômago e objetos que podem provocar obstruções gastrointestinais graves (Ferreira et al., 2007).

A falta de literatura especializada sobre seus hábitos de vida faz com que sejam extrapolados manejos de outros animais. Com isso, ocorrem equívocos sobre a alimentação, que é um dos principais problemas apresentados por esta espécie (Proença \& Mayer, 2014). Os coelhos têm o hábito de se lamber e ingerir os pelos. Entretanto, estes animais não têm capacidade de vomitá-los, o que se torna um problema quando a ingesta é alta e a dieta inadequada, pois estes podem atuar como corpos estranhos gástricos e levar à obstrução (Fukumura et al., 2012).

A estase e a obstrução gastrointestinal são condições frequentes em coelhos podendo ser primárias, ou estarem associadas a outros fatores, como dietas pobres em fibras e de baixa digestibilidade que levam à diminuição da motilidade intestinal (Krempels et al., 2000; Yorston, 2013). Outras causas são corpos estranhos, alterações na dieta, desidratação, estresse, obesidade, sedentarismo e uso medicamentos como antibióticos, anti-inflamatórios e opióides (Huynh et al., 2014; Prebble, 2012). Os sinais clínicos mais comuns são inapetência, anorexia, desidratação, trismo mandibular, desconforto e distensão abdominal, redução na quantidade e tamanho dos bolos fecais ou ausência de defecação (DeCubellis \& Graham, 2013). O diagnóstico pode ser realizado por meio de anamnese detalhada, achados do exame físico e confirmação com exame radiográfico (Yorston, 2013).

Devido ao aumento do número de atendimentos a coelhos nas clínicas veterinárias e a falta de literatura especializada, além da alta prevalência da enfermidade, o objetivo do presente estudo foi abordar sobre a condição de estase e obstrução gastrointestinal em coelhos domésticos.

\section{A origem do coelho doméstico}

O coelho doméstico descende do coelho europeu silvestre; porém, se trata da mesma espécie. Ele é pertencente à Ordem Lagomorpha, que inclui duas famílias, sendo elas a Ochotonidae, que engloba as espécies dos pikas e a Leporidae, que contém 11 gêneros e mais de 50 espécies de coelhos e lebres (Crowell-Davis, 2007). Nem sempre sua taxonomia foi assim relatada, pois até em meados do século $\mathrm{XX}$, os lagomorfos eram considerados subordem da Ordem Rodentia, contudo, estes não partilham semelhanças fundamentais, como nas dentições e nos padrões de mastigação dos roedores, tendo maiores semelhanças com a ordem Artiodactyla (mamíferos ungulados). Sendo assim, levou-se a crer que eles evoluíram de um mamífero herbívoro ancestral comum, também parente do cavalo (Crossley, 2003; Crowell-Davis, 2007).

Os exemplares de coelhos (Figura 1) têm sido encontrados em vários tamanhos, cores, pelagens e raças, e são resultados de cruzamentos na tentativa de cumprir seus objetivos, seja para o fornecimento de carne, lã, pêlos, experimentação animal, exposição ou companhia (Crossley, 2003; Irving-Pease et al., 2018).

\section{Comportamento e manejo}

Coelhos são presas, por isso, são animais "desconfiados" por natureza, e podem ser receosos em ambientes e situações novas, pois seus ancestrais dependiam de um estado de alerta e rapidez ao lidar com situações que pudessem apresentar risco (Harcourt-Brown \& Harcourt-Brown, 2002). Devido a essa necessidade de estar em estado de alerta constantemente, os coelhos se apoiam em seus membros posteriores, os quais apresentam a musculatura muito desenvolvida, para visualizar e avaliar melhor a 
área em que ele se encontra, cheirar, ouvir e pedir guloseimas. Seus sentidos são apurados, por isso, é importante em seu manejo, evitar barulhos altos e movimentos bruscos (Bradley, 2000; Ness, 2002).

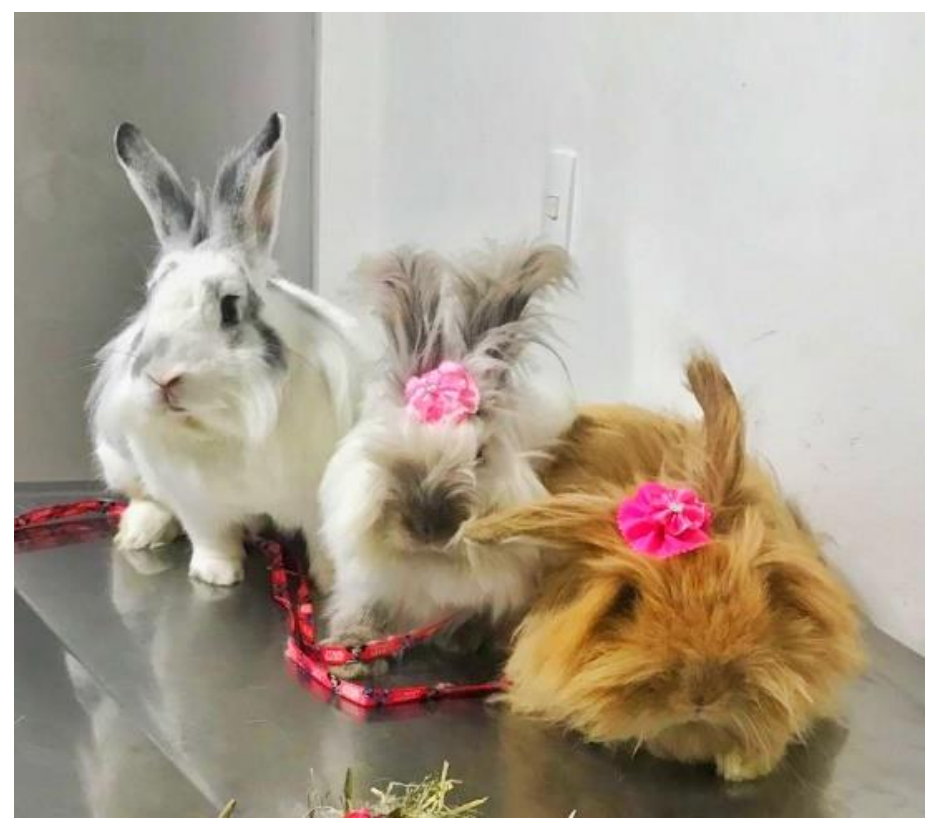

Figura 1. Coelhos domésticos representados em diferentes raças (Borboleta Ingles/Lionhead, Angorá Inglês e Lionhead) e cores (branco e cinza, branco com extremidades escuras e caramelo).

Os coelhos são animais sociáveis, tanto com outros de sua espécie, como com humanos e animais de estimação. São animais perspicazes e que necessitam de atenção e estímulos por parte dos tutores (Bradley, 2000). Seus hábitos de higiene são rígidos, tanto os próprios quanto para com os outros indivíduos do grupo (Meredith \& Redrobe, 2002). Eles tendem a urinar e defecar em um local definido, o que favorece o aprendizado do uso da liteira, como os gatos. Durante o dia podem descansar em decúbito lateral, ventral ou até dorsal, em posição de conforto e relaxamento (Bradley, 2000). Contudo, podem se tornar hostis e agressivos quando se sentem assustados ou com dor e demonstrar a agressividade por meio de vocalização e ataque. De forma geral, segue-se a sequência: grunhido-ataquemordida (Bradley, 2000; Meredith \& Redrobe, 2002). Os sinais clínicos de dor são difíceis de serem notados e qualquer mudança de comportamento pode ser o primeiro indicador de alguma anormalidade (Bradley, 2000; Crowell-Davis, 2007).

\section{Comportamento alimentar}

Entender as particularidades digestivas do coelho, e em como os componentes alimentares são digeridos, é necessário para que o animal tenha boa saúde (Harcourt-Brown \& Harcourt-Brown, 2002). O coelho é um animal herbívoro não ruminante (Gidenne, 2003; Kahn et al., 2005) que possui um estômago considerado pequeno, um ceco complexo e um rápido trânsito digestivo (Ferreira et al., 2007; Harcourt-Brown \& Harcourt-Brown, 2002). Seu organismo tem elevada taxa metabólica, sendo necessária que sua dieta tenha alta concentração energética. (Kahn et al., 2005). Um coelho adulto alimenta-se de 20 a 40 vezes ao dia, sendo essa ingesta permanente, importante para a manutenção do trânsito gastrointestinal (Ferreira et al., 2007). Dentro os segmentos digestivos, o ceco ocupa de 40 a $60 \%$ do trato digestivo, o estômago é responsável por apenas $15 \%$ do volume total. A região da cárdia é bem desenvolvida, impedindo-o de vomitar (Harcourt-Brown \& Harcourt-Brown, 2002). A digestão dos coelhos é similar à da maioria das espécies monogástricas até o intestino grosso, e a partir da passagem do bolo alimentar para esta porção do trato digestivo, eles apresentam fisiologia digestiva muito particular, diferente de outras espécies de herbívoros. No intestino grosso ocorre a fermentação cecal, a excreção seletiva de fibra, o que permite a formação de cecotrofos e a reingesta do material fecal, chamada cecotrofia. Os cecotrofos são compostos por aminoácidos, ácidos graxos voláteis, micro-organismos e vitaminas do complexo B e K (Ferreira et al., 2007; Harcourt-Brown \& HarcourtBrown, 2002). 


\section{Estase gastrointestinal}

O termo estase gastrointestinal, é utilizado para definir a diminuição da motilidade gastrointestinal levando a um quadro de disbiose (Fisher, 2010; Reusch, 2005) Sua etiologia é incerta, sendo raramente um problema primário (Krempels et al., 2000; Lichtenberger \& Lennox, 2010). Dentre suas causas relatadas mais frequentemente estão a anorexia, desidratação, problemas dentários, dieta inadequada, alterações bruscas na dieta, alguns fármacos, obesidade e ingestão de corpos estranhos, dor, doenças sistêmicas, doença uterina, infecção urinária, fatores ambientais gerando stress como brigas por dominância, presença de predadores, perda de um companheiro, mudança de rotina ou dieta (Fisher, 2010; Reusch, 2005). Como sinais clínicos, o animal apresenta inapetência, anorexia, desidratação, trismo mandibular, desconforto e distensão abdominal, redução na quantidade e tamanho dos bolos fecais ou ausência de defecação, cecotrofos não ingeridos, presença de diarreia com ou sem muco, sialorreia (Figura 2) (DeCubellis \& Graham, 2013; Lichtenberger \& Lennox, 2010).
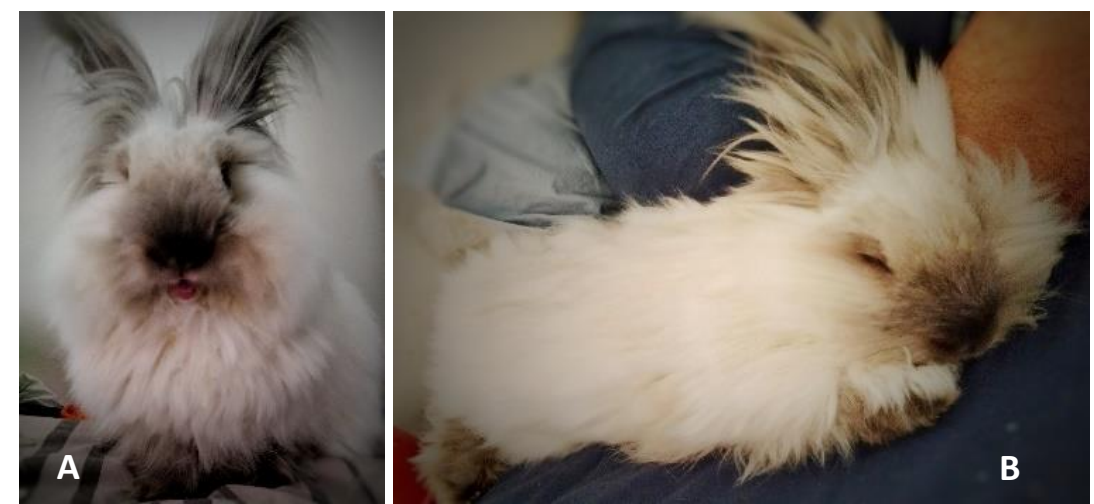

Figura 2. A: Coelho doméstico com sialorreia devido à estase gastrointestinal.

B: Coelho doméstico em posição antiálgica devido ao desconforto.

Em animais idosos e de meia idade, ocorre com maior frequência, geralmente interligada a uma dieta inapropriada, que inclui guloseimas, cereais, apenas ração comercial e pouca quantidade de fibra (Fisher, 2010; Harcourt-Brown \& Harcourt-Brown, 2002; Oglesbee, 2006). Os achados durante o exame físico incluem desidratação, aumento gástrico e intestinal, distensão abdominal, podendo ocorrer timpanismo gástrico e choque. Caso a causa primária seja uma infecção, pode haver um quadro febril. À palpação, o ceco poderá estar repleto de gás, fluidos ou conteúdo e o estomago dilatado (Paul-Murphy, 2007).

\section{Obstrução gastrointestinal}

A obstrução gastrointestinal ocorre devido à ingestão de corpos estranhos, como tecidos, tapetes, maior quantidade de pêlos, pequenos objetos, e também pode ser causado por doenças inflamatórias, tumores, parasitos ou invaginações na porção intestinal, cistos e adesões pós-cirúrgicas (HarcourtBrown \& Harcourt-Brown, 2002). É uma doença de aparecimento súbito, aguda e de rápida progressão. Os animais se apresentam com o abdômen dilatado e o estômago timpanizado com alta quantidade de saliva, pois por não terem a capacidade de vomitar, esta se acumula no estômago, ocasionando dilatação e podendo ocorrer sua ruptura (Harcourt-Brown \& Harcourt-Brown, 2002; Lichtenberger, 2005; Oglesbee, 2006).

Coelhos que apresentam obstrução gastrointestinal geralmente chegam ao médico veterinário em choque hipovolêmico. Dentre os sinais clínicos apresentados estão anorexia, diminuição ou interrupção da produção de pellets fecais, depressão, desidratação, dor abdominal, timpanismo gástrico, dentre outros e podem vir a óbito rapidamente, dentro de 24 a 48 horas (Prebble, 2012).

\section{Diagnóstico e prognóstico}

Uma das partes mais importantes do diagnóstico é a diferenciação entre estase e obstrução, e isso deve ser feito de forma mais rápida possível, pois na estase opta-se pelo uso de fármacos, enquanto o tratamento definitivo da obstrução é cirúrgico (Harcourt-Brown \& Harcourt-Brown, 2002; Lichtenberger, 2005; Oglesbee, 2006). É de suma importância que aos primeiros sinais de obstrução 
gastrointestinal, se inicie imediatamente a terapêutica adequada, e que se utilizem formas complementares de diagnóstico para a pesquisa da causa primária. Quanto mais demorado é o estabelecimento do diagnóstico e da terapia adequada, mais riscos o paciente pode estar exposto (Fisher, 2010; Lichtenberger \& Ko, 2007).

Para animais que não toleram o manejo, deve-se utilizar a sedação, que são benéficas para a diminuição do estresse e da ansiedade, bem como o alivio da dor (Capello \& Lennox, 2008; Fisher, 2010). Os medicamentos mais utilizados são o midazolam isolado ou associado a um opioide (Lichtenberger \& Ko, 2007). Outros fármacos são a acepromazina, medetomidina, fentanil ou a associação de acepromazina com butorfanol (Flecknell, 2008; Harcourt-Brown \& Harcourt-Brown, 2002).

A coleta de sangue para análise deve ser realizada nas veias cefálica, jugular, safena lateral, central e marginal da orelha, sendo a ideal, dentre as opções, a safena lateral (Graham, 2006; Sjoberg \& Odberg, 2003). As análises sanguíneas ajudam na detecção de alterações como hipoproteinemia e anemia, estado de desidratação (Harcourt-Brown \& Harcourt-Brown, 2002). Outro exame importante é a radiografia, sendo o exame de escolha para o diagnóstico, auxiliando na diferenciação entre estase e obstrução gastrointestinal (Girling, 2003; Harcourt-Brown \& Harcourt-Brown, 2002). No caso do tratamento da estase, devem ser realizadas radiografias a cada 3-4 horas, ajudando na determinação da eficácia terapêutica e em pacientes mais estáveis, devem ser realizadas a cada 24 horas (Lichtenberger \& Lennox, 2010). Os sinais apontados na radiografia quando o animal apresenta estase gastrointestinal incluem bolsas de gás em todo o trato gastrointestinal e o estômago arredondado devido ao acúmulo de conteúdo, podendo o ceco também estar impactado ou distendido com gás (Oglesbee, 2006). Os sinais apontados na radiografia quando o animal apresenta obstrução gastrointestinal parcial ou total incluem estômago arredondado, preenchido com fluidos ou gás, onde o gás pode estar distalmente a zona obstruída, devido a uma estase secundaria (Figura 3) (Lichtenberger \& Lennox, 2010).

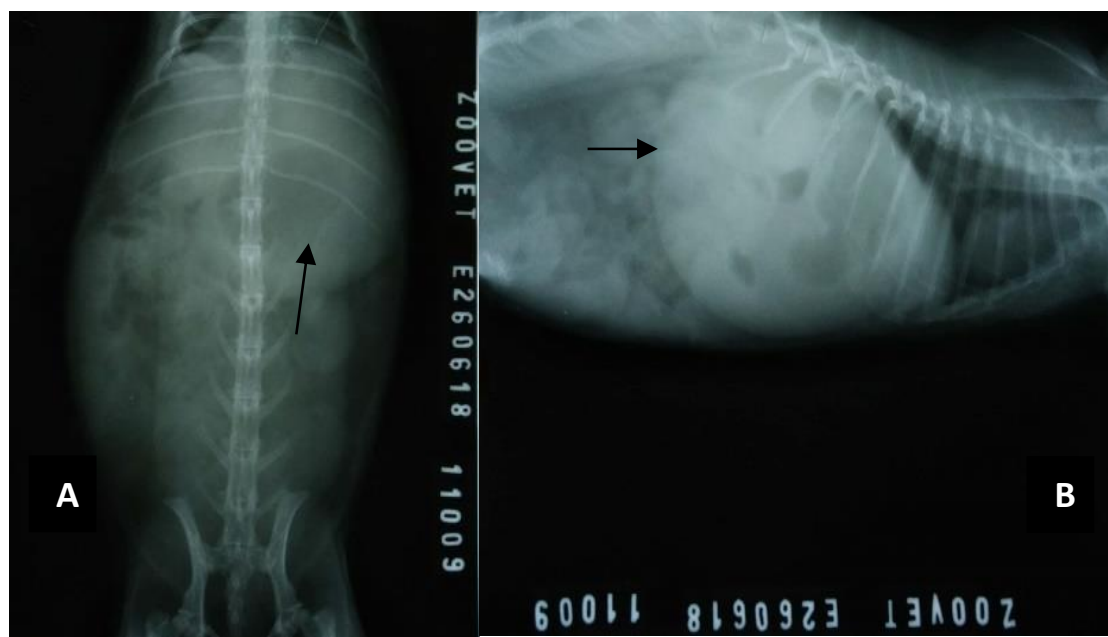

Figura 3. Radiografias abdominais simples de região de abdômen, nas projeções ventrodorsal (A) e latero-lateral (B) de paciente da espécie cunícula que apresentava quadro de diagnóstico de obstrução gastrointestinal. Notar o quadro de dilatação estomacal com presença de material radiopaco difusamente presente (setas).

O prognóstico da estase e da obstrução gastrointestinal depende da causa primária, bem como da rapidez em diagnosticá-la e instituir o tratamento adequado, uma vez que, em casos avançados, os animais dão entrada na clínica com sinais de choque circulatório, membros frios, TPC aumentado, além de taquicardia. Quando iniciado precocemente, o prognostico da estase gastrointestinal é de excelente a bom (Oglesbee, 2006). Já o prognóstico pós-cirúrgico da obstrução gastrointestinal, é considerado ruim (Harcourt-Brown \& Harcourt-Brown, 2002; Oglesbee, 2006).

\section{Tratamento e profilaxia}

Tanto a estase quanto a obstrução gastrointestinal afetam a hidratação do paciente, por isso, a primeira ação a ser tomada é a fluidoterapia, utilizando fluido cristaloide (Harcourt-Brown \& Harcourt- 
Brown, 2002; Lichtenberger \& Lennox, 2010). Pode esta ser realizada por via intraóssea ou intravenosa, sendo a via subcutânea não indicada, devido à sua lenta absorção e sua baixa perfusão periférica (Harcourt-Brown \& Harcourt-Brown, 2002; Oglesbee, 2006), sendo esta via indicada apenas para pacientes já estabilizados. A hidratação intra-oral somente é indicada quando não há suspeita de obstrução (Lichtenberger \& Lennox, 2010; Oglesbee, 2006). Além do fluído, pode ser necessário administrar soluções especificas com o intuito de corrigir os déficits (Lichtenberger, 2005). Os coelhos com estase e obstrução gastrointestinal podem apresentar-se em estado de choque, com hipotensão, bradicardia, hipotermia, pulso fraco e mucosas hipocoradas, sendo necessário que o médico veterinário reconheça e identifique o choque, realizando uma combinação de cristaloides e coloides, evitando assim uma acumulação de fluidos nos pulmões e pleura e o imediato aquecimento do paciente (Fisher, 2010; Graham, 2006; Lichtenberger \& Lennox, 2010).

Como o animal com estase gastrointestinal se apresenta anoréxico, é necessário que se providencie um suporte nutricional eficiente. As falhas na suplementação do animal podem levar ao quadro de lipidose hepática, com consequente piora do quadro do paciente. A alimentação deve ser fornecida com o auxílio de uma seringa e tendo precaução para que não seja aspirado. Quando há uma recusa do animal, deve ser realizada via tubo nasogástrico (Graham, 2006; Harcourt-Brown \& Harcourt-Brown, 2002). Além da alimentação forçada, é necessário deixar à disposição do paciente, feno, vegetais frescos e ervas aromática que atuam como estimulantes do apetite (Lichtenberger \& Lennox, 2010; Reusch, 2005).

O tratamento cirúrgico somente é realizado quando há uma suspeita verdadeira de obstrução gastrointestinal, pois a estase gastrointestinal, geralmente é resolvida através de tratamento medicamentoso (Harcourt-Brown \& Harcourt-Brown, 2002; Oglesbee, 2006). O objetivo do tratamento é restaurar o apetite do animal, corrigir os déficits eletrolíticos, corrigir a desidratação, estimular o esvaziamento gástrico, eliminar a ingesta impactada e retornar à motilidade gastrointestinal normal (Harcourt-Brown \& Harcourt-Brown, 2002; Oglesbee, 2006). É necessário que seja realizado o controle da dor, por isso a analgesia tem um papel fundamental no tratamento. São utilizadas três classes de medicamentos, sendo eles os opioides, AINEs e antagonistas dos receptores N-metil-D-Aspartato (quetamina) (Barter, 2011). O opioide mais utilizado é o butorfanol, sendo um ótimo analgésico visceral, podendo ser utilizado em combinação com a quetamina, em doses baixas, com cristaloide em uma bomba infusora, sem se verificar efeitos indesejados (Lichtenberger \& Ko, 2007). O AINE mais usado em coelhos é o meloxicam, tendo uma boa tolerância, também pode ser usado o carprofeno, ou a flunixina meglumina, mas tendo que ter cuidado com seus efeitos secundários, como alteração da função renal, erosão gastrointestinal e ausência da produção de cecotrofos (Harcourt-Brown \& HarcourtBrown, 2002; Lichtenberger \& Ko, 2007). A avaliação da resposta aos analgésicos é realizada com a observação da volta ao comportamento normal, comendo, bebendo, defecando e realizando auto-higiene (Bradley, 2001).

Quando há estase gastrointestinal, ocorre a necessidade de promover a motilidade gastrointestinal com o uso de fármacos procinéticos, sendo os mais utilizados a metoclopramida e a cisaprida (Lichtenberger \& Lennox, 2010). Elas podem ser utilizadas em conjunto, uma vez que atuam em diferentes áreas, sendo a metoclopramida no trato gastrointestinal superior e a cisaprida, primariamente, no inferior. Contudo, em caso de suspeita de obstrução gastrointestinal, os fármacos pró-cinéticos são contraindicados (Lichtenberger \& Lennox, 2010). Como o trato gastrointestinal está repleto de gás, deve ser administrado antiflatulentos, como a dimeticona e a simeticona, que atuam realizando a alteração da superfície de tensão das bolhas de gás no intestino, tornando-as mais facilmente eliminadas. O encorajamento do animal a realizar exercícios físicos, caso este não esteja muito debilitado, e a realização de massagem é uma das formas mais simples e estimular o peristaltismo intestinal e a fragmentar os conteúdos impactados no estomago (Fisher, 2010).

Pelo explicitado, a profilaxia é considerada o melhor recurso para as condições de estase e obstrução gastrointestinal. O manejo alimentar deve ser regrado (Figura 4), à base de fibra de cadeia longa e indigerível, por meio do fornecimento de feno e de gramíneas à vontade. Quando oferecida ração peletizada, esta deve garantir que contenha altos níveis de fibra (no mínimo $18 \%$ ), baixo teor de amido (de 4 a $5 \%$ ), e adequada concentração de proteínas (de 12 a $14 \%$ ), devendo ser oferecida de $25 \mathrm{~g} / \mathrm{kg} / \mathrm{dia}$ (Gidenne, 2010; Irlbeck, 2001). Para que o coelho se mantenha hidratado, deve ter água fresca e limpa à vontade, e também verduras frescas como chicória, folhas de cenoura, agrião, folhas de rabanete, folha 
de bananeira, salsa, tendo cuidado com alguns vegetais, como o dente de leão que em excesso leva a uma condição chamada água vermelha, uma queixa renal, folhas de repolho que contém glucosinolato prejudicando a absorção do iodo pela tireoide, a alface que contém lactucarium, um soporífico que tem ação semelhante ao ópio sendo danoso à saúde do animal, assim como plantas tóxicas, as quais os coelhos domésticos não diferenciam e podem ingerir caso estejam ao seu alcance, e raízes e frutas devem ser dadas com moderação devido ao seu alto teor de açúcares (Bradley, 2001; Lowe, 2010).

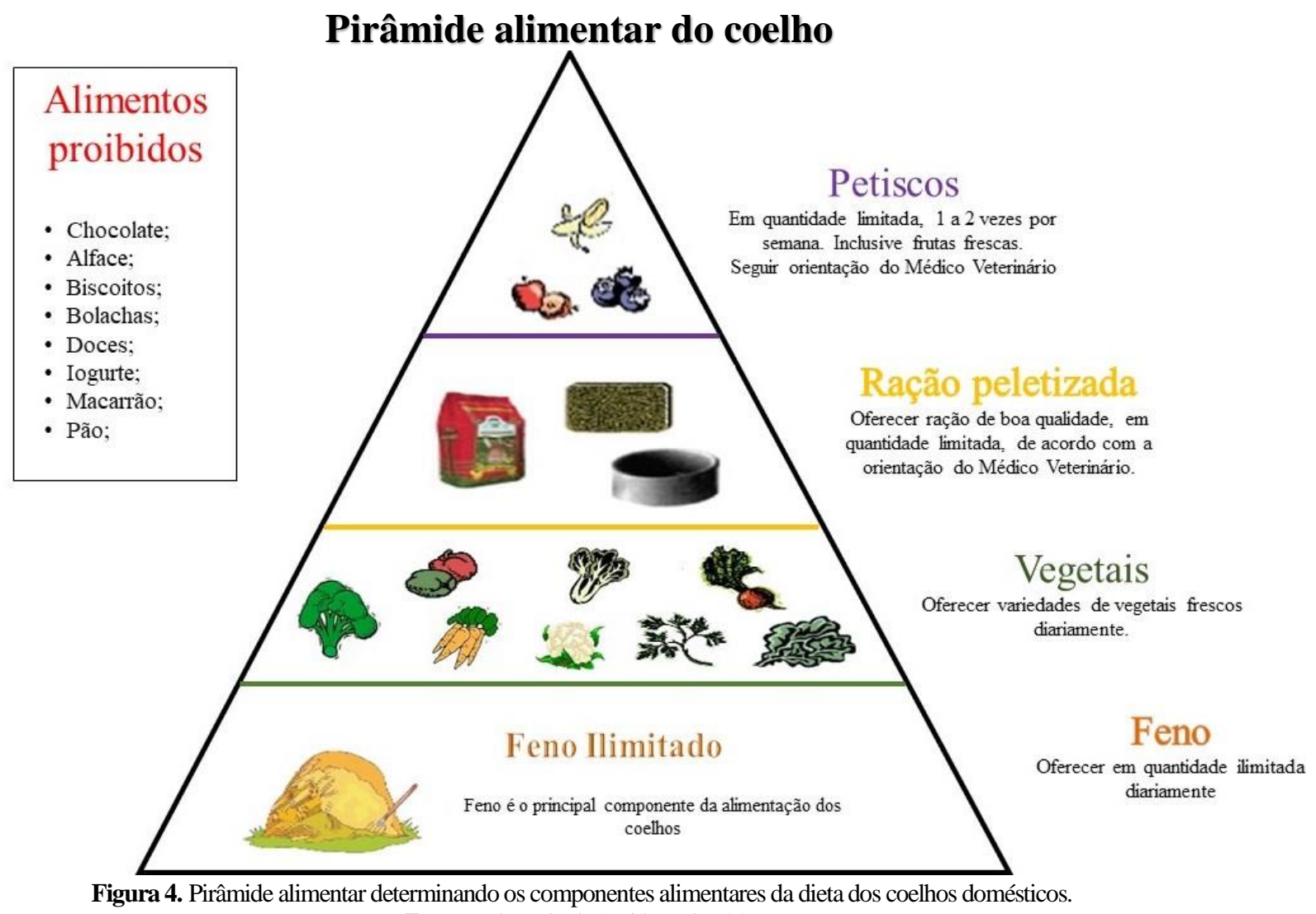

Fonte: Adaptado de Smith et al., 2009

O coelho deve ficar em local com espaço suficiente para realizar exercício físico regular, realizar escovação diariamente e mantê-lo longe de fontes de estresse (Kahn et al., 2005; Oglesbee, 2006). Fêmeas devem ser submetidas à esterilização como forma de prevenção, pois além da probabilidade de desenvolverem neoplasias reprodutivas e mamárias, podem desenvolver pseudociese, muitas das vezes sendo causa da estase e obstrução gastrointestinal. Visitas de rotina ao médico veterinário são importantes, uma vez que podem ser diagnosticados problemas que podem levar à estase e obstrução gastrointestinal, como por exemplo, os problemas dentários (Bradley, 2001).

\section{Considerações finais}

Os coelhos são animais considerados presas, os coelhos têm o comportamento de se esconder quando há algum problema, por isso, à mínima mudança de comportamento, é de importante que seja levado a um profissional para lidar com o problema apresentado e instituir o tratamento adequado o mais rápido possível, a fim de minimizar os riscos.

Dentre as enfermidades apresentadas por essa espécie, a estase e a obstrução gastrointestinal são consideradas de alta prevalência. Os fatores que podem levar ao quadro de estase e obstrução gastrointestinal são diversos e comuns na clínica de animais exóticos, por isso o médico veterinário deve utilizar de artifícios como exames complementares para realizar o diagnóstico, como radiografia e análise sanguínea. Tendo em vista que são doenças que debilitam o animal e podem levar até mesmo ao óbito, o mais importante é realizar medidas de prevenção, como manejo alimentar adequado, à base de muita fibra, ração comercial de qualidade, água e verduras frescas, além de um ambiente amplo o 
suficiente para o animal se exercitar, escovação diária, esterilização e visitas periódicas ao médico veterinário, gerando bem-estar e qualidade de vida ao coelho.

O médico veterinário tem a responsabilidade de educar o cliente sobre a importância do manejo alimentar e ambiental dos coelhos, pois a prevenção juntamente com a detecção precoce dos sinais clínicos, tem um papel importante no tratamento da doença. No caso desta, quanto mais extenso o tempo, pior será o prognóstico.

\section{Referências bibliográficas}

Barter, L. S. (2011). Rabbit analgesia. Veterinary Clinics: Exotic Animal Practice, 14(1):93-104.

Bradley, T. (2000). Rabbits: understanding normal behavior. Exotic DVM, 2(1):19-24.

Bradley, T. A. (2001). What every veterinarian needs to know about rabbits. Exotic DVM, 3(1):42-46.

Capello, V. \& Lennox, A. (2008). Rabbit. In V. Capello \& A. Lennox (Eds.), Clinical radiology of exotic companion mammals. Iowa, EUA.

Crossley, D. A. (2003). Oral biology and disorders of lagomorphs. Veterinary Clinics: Exotic Animal Practice, 6(3):629-659.

Crowell-Davis, S. L. (2007). Behavior problems in pet rabbits. Journal of Exotic Pet Medicine, 16(1):3844.

DeCubellis, J. \& Graham, J. (2013). Gastrointestinal disease in guinea pigs and rabbits. Veterinary Clinics: Exotic Animal Practice, 16(2):421-435.

Ferreira, M. P., Alievi, M. M., Beck, C. A. C., Silva, A. d. P. F., Santos Júnior, E. B. S., Beheregaray, W., . . Gonzalez, P. C. S. (2007). Corpo estranho gástrico em um coelho (Oryctolagus cuniculus). Acta Scientiae Veterinariae, 35(2):249-251.

Fisher, P. G. (2010). Standards of care in the 21st century: the rabbit. Journal of Exotic Pet Medicine, 19(1):22-35.

Flecknell, P. A. (2008). Analgesia from a veterinary perspective. British Journal of Anaesthesia, 101(1):121-124. doi: https://doi.org/10.1093/bja/aen087.

Fukumura, K., Haneda, R., Endoh, T., Takano, M., Mizoguchi, Y., Matsuoka, T. \& Asano, Y. (2012). Gastric hairballs in rabbits: Significance in developmental toxicity study. Congenital Anomalies, 52(2):104-105.

Gidenne, T. (2003). Fibres in rabbit feeding for digestive troubles prevention: respective role of lowdigested and digestible fibre. Livestock Production Science, 81(2-3):105-117.

Gidenne, T. (2010). Recent advances in rabbit nutrition: emphasis on fibre requirements. A review. World Rabbit Science, 8(1):23-32.

Girling, S. (2003). Veterinary Nursing of Exotic Species. USA: Blackwell publishing.

Graham, J. (2006). Common procedures in rabbits. Veterinary Clinics: Exotic Animal Practice, 9(2):367-388.

Harcourt-Brown, F. \& Harcourt-Brown, N. H. (2002). Textbook of rabbit medicine: ButterworthHeinemann.

Huynh, M., Vilmouth, S., Gonzalez, M. S., Carrasco, D. C., Di Girolamo, N. \& Forbes, N. A. (2014). Retrospective cohort study of gastrointestinal stasis in pet rabbits. Veterinary Record, 175(9):225225.

Irlbeck, N. A. (2001). How to feed the rabbit (Oryctolagus cuniculus) gastrointestinal tract. Journal of Animal Science, 79(Suppl.):E343-E346.

Irving-Pease, E. K., Frantz, L. A. F., Sykes, N., Callou, C. \& Larson, G. (2018). Rabbits and the specious origins of domestication. Trends in Ecology \& Evolution, 33(3):149-152.

Kahn, C. M., Line, S. \& Aiello, S. (2005). The merck veterinary manual. Merck \& Co. USA: Whitehouse Station.

Krempels, D., Cotter, M. \& Stanzione, G. (2000). Ileus in domestic rabbits. Exotic DVM, 2(4):19-21. 
Lichtenberger, M. (2005). Fluid resuscitation and nutritional support in rabbits with gastric stasis or gastrointestinal obstruction. Exotic DVM, 7(2):34-38.

Lichtenberger, M. \& Ko, J. (2007). Critical care monitoring. Veterinary Clinics of North America: Exotic Animal Practice, 10(2):317-344.

Lichtenberger, M. \& Lennox, A. (2010). Updates and advanced therapies for gastrointestinal stasis in rabbits. Veterinary Clinics: Exotic Animal Practice, 13(3):525-541.

Lowe, J. A. (2010). Pet rabbit feeding and nutrition. In C. Blas \& J. Wiseman (Eds.), Nutrition of the rabbit (pp. 294). Madri, Espanha.

Meredith, A. \& Redrobe, S. (2002). BSAVA manual of exotic pets. London, UK: British Small Animal Veterinary Association.

Ness, R. D. (2002). Alternatives for the anxious patient. Exotic DVM, 31-12.

Oglesbee, B. L. (2006). The 5-minute veterinary consult: ferret and rabbit: Blackwell Ames.

Paul-Murphy, J. (2007). Critical care of the rabbit. Veterinary Clinics of North America: Exotic Animal Practice, 10(2):437-461.

Prebble, J. (2012). Gastrointestinal stasis and obstructive Ileus in the rabbit. The Veterinary Nurse, 3(6):366-372.

Proença, L. M. \& Mayer, J. (2014). Prescription diets for rabbits. Veterinary Clinics: Exotic Animal Practice, 17(3):485-502.

Reusch, B. (2005). Rabbit gastroenterology. Veterinary Clinics: Exotic Animal Practice, 8(2):351-375.

Sjoberg, J. G. \& Odberg, E. (2003). One perspective on selected blood collection sites in exotic species. Exotic DVM, 5(4):27-32.

Smith, M. H., Meehan, C. L., Ma, J. M., Hisakawa, N., Dasher, H. S., Camarillo, J. D. \& Techanun, J. (2009). Rabbit Nutrition: What you need to know. Rabbits: From the point of View, University of Calofornia Agriculture and Natural Resources, 3,1-27.

Yorston, M. (2013). Gastrointestinal stasis in Rabbits. New Zealand Veterinary Nurse, 1926-29.

Recebido: 11 de outubro, 2019.

Aprovado: 05 de novembro, 2019.

Publicado: 17 de dezembro, 2019.

Licenciamento: Este artigo é publicado na modalidade acesso aberto sob a licença Creative Commons Atribuição 4.0 (CC-BY 4.0), a qual permite uso irrestrito, distribuição, reprodução em qualquer meio, desde que o autor e a fonte sejam devidamente creditados. 